\title{
IMPACTO DE LA RELACIÓN PROVEEDOR - CLIENTE EN EL DESEMPEÑO DE PEQUEÑOS Y MEDIANOS CONTRATISTAS DE BAHÍA BLANCA. \\ Una aproximación econométrica
}

García Casal Irene*

Leonardi, Viviana ${ }^{* *}$

\begin{abstract}
Resumen
Los crecientes niveles de competitividad mundial han llevado a las grandes firmas a operar con mayor grado de flexibilidad. Entre las estrategias desplegadas, la más importante ha sido la creación de redes de empresas, que unen unidades productivas grandes y pequeñas operando en diferentes sectores, regiones e incluso países. Este proceso de descentralización productiva ha permitido a las pymes recuperar un rol protagónico, que parecía perdido durante la vigencia del paradigma fordista.El objetivo central del presente trabajo es analizar, a través de un modelo econométrico, la importancia que reviste para una pequeña y mediana empresa ser proveedor de una gran planta que manufactura commodities, ambas radicadas en la ciudad de Bahía Blanca.
\end{abstract}

Palabras clave: economía industrial - Pymes - redes

\section{Abstract}

The growing levels of world competitiveness have taken to big corporations to work with more degree of flexibility. Among the deployed strategies, the most important has been the creation of networks that join big and small productive units operating in different sectors, regions and even countries. This process of productive decentralization has allowed medium and small firms to recover a protagonistic role that seemed to be lost during the validity of the fordist paradigm.The central objective of the present work is to analyze, through an econometric model, the importance for a small and medium company to be a supplier of a great plant that manufactures commodities, both established in Bahía Blanca’s city.

Keywords: industrial economy - Smes - networks

\section{INTRODUCCION}

El trauma que produjo la crisis económica mundial de los años 70 y principios de los 80 , planteó a las grandes empresas la necesidad de reorganizar sus relaciones externas y reestructurar su funcionamiento interno. Así, en lugar de disminuir el poder económico concentrado en ellas, lo que se visualiza es un cambio de forma de dicha concentración de poder vía alianzas estratégicas, acuerdos tecnológicos y financieros de corto y largo plazo entre las mismas empresas, con los gobiernos a todos los niveles y con legiones de pequeñas y medianas firmas que actúan como proveedores y subcontratistas.

La producción está siendo cada vez más descentralizada, dado que los directivos intentan aumentar su flexibilidad para hacer frente a las inmensas barreras de entrada a los mercados y a la

\footnotetext{
* Departamento de Economía, Universidad Nacional del Sur

** Departamento de Economía, Universidad Nacional del Sur
} 
incertidumbre crónica propia de las condiciones políticas y de las diferentes demandas de los consumidores.

De todas las reacciones y prácticas llevadas a cabo por las grandes firmas, la más importante, por las consecuencias que ha reportado, ha sido la creación de redes de empresas, que unen unidades productivas grandes y pequeñas operando en diferentes sectores, regiones e incluso países.

Walter Powell (1990), una de las figuras más representativa de la teoría norteamericana sobre redes de producción como forma de organización industrial, sostiene que las redes pueden llegar a convertirse en la "firma institucional" de nuestra era dado que ofrecen a los directivos la mejor solución operativa a los retos impuestos por la creciente necesidad de flexibilidad. Para Powell, las formas de red facilitan la entrada en los negocios de aspectos tan positivos como pueden ser la reciprocidad, el altruismo, la amistad, la reputación y la colaboración, que actuarán como principios de gobierno frente al desmedido interés propio en el caso del libre mercado y de la administración central para las corporaciones integradas verticalmente.

Si bien desde el punto de vista de la teoría de Williamson (1985), la estructura en forma de red incrementa los costos de transacción, las redes como forma organizacional siguen proliferando. Aparentemente " las redes son algo más que las sumas de sus partes"; existe algo parecido a las externalidades de la red” ( DeBresson y Amesse, 1991)

De los distintos tipos de redes de producción (industrias tipo taller, polos industriales basados en pequeñas empresas, sistemas de producción limitados geográficamente y basados en grandes empresas, alianzas estratégicas) nos interesa analizar el caso de los sistemas de producción limitados geográficamente y basados en grandes empresas, ya que es la formación que más se asemeja al caso práctico que vamos estudiar.

El objetivo central del presente trabajo es investigar a través de un modelo econométrico la importancia que reviste para una pequeña y mediana empresa ser proveedor de una planta grande que manufactura commodities. Para ello se ha planteado un modelo con variable endógena dicotómica y cualitativa (probit) utilizando el método econométrico de máxima verosimilitud con el soporte del software STATA. Dicho análisis se realizó a partir de datos relevados en el año 2002 a una muestra de ciento once firmas pequeñas y medianas proveedoras de grandes empresas localizadas en Bahía Blanca.

1. Algunas consideraciones respecto a la vinculación económica entre grandes plantas industriales y pequeñas empresas

No existe ninguna economía a nivel mundial que dependa, tan extensivamente, de las redes de producción como lo hace la japonesa. De hecho, los modelos de comportamiento japoneses han dado al resto del mundo un instrumental con el que entender cómo el poder económico concentrado puede conciliarse con la producción descentralizada y transfronteriza de forma que se promocionen el aprendizaje organizacional, la innovación sistemática y la flexibilidad frente a las presiones competitivas.

Los ejemplos actuales más importantes de sistemas de producción complejos dispuestos en forma de red son los "keiretsu de oferta" japoneses, como Toyota, Sony y NEC, presentes en especial en la industria automovilística, la de la electrónica de consumo y la maquinaria, donde el producto final está conformado por una multitud de componentes que se organizan alrededor de empresas matrices con un elemento dominante en la función de ensamblaje/montaje final que generan una densa red de pequeños y medianos proveedores. Este proceso de descentralización productiva ha permitido 
a las pymes recuperar un rol protagónico que parecía perdido en los tiempos de la producción masiva y a gran escala características del paradigma fordista.

En la forma de vinculación “a la japonesa”, la casa matriz suministra asistencia técnica y financiera a por lo menos el primer estrato de proveedores, estableciéndose una continua cooperación en la ingeniería de procesos y productos, investigación común en realizaciones tecnológicas y continuo intercambio de información. Así, los compradores seleccionan a los proveedores en función de la calidad, el nivel y la extensión de los servicios, el contenido tecnológico y la capacidad de integración. Se establecen contratos de suministro de largo plazo en donde predominan las consideraciones de calidad y fluidez de información, aspectos esenciales para las operaciones bajo los modernos métodos organizacionales como los sistemas de control de inventarios just-in time o los sistemas de gestión de calidad total. Bourgault, M., Lefebvre, L. y Lefebvre, E. (1995), a través de un análisis econométrico, encuentran que los esfuerzos por establecer vínculos estables entre los grandes establecimientos y sus proveedores y subcontratistas tienen un impacto positivo en el desempeño de estos últimos.

En Occidente, la relación estilizada inter-firma adquiere otras características. Los empresarios negocian sobre una garantía de mínimo nivel de calidad, las demandas son órdenes simples de corto plazo, que son testeadas sistemáticamente para una satisfactoria provisión. Las prácticas de abastecimiento son generalmente adversarias más que cooperativas y las vinculaciones están manejadas principalmente por consideraciones de costos, dado un mínimo requerimiento de calidad. De ello resulta que la competencia entre los diversos proveedores está guiada por precios. Tampoco se observa un intercambio de información ni compromisos conjuntos para desarrollar actividades de investigación y desarrollo (De Toni, A.; Nassimbeni, G., Tonchia, S., 1995; y Nooteboom, 1995).

Este comportamiento es propio, fundamentalmente, de las empresas norteamericanas. Es cierto que algunas de ellas ya experimentan con alianzas estratégicas, fabrican en colaboración con los proveedores y ponen en práctica otras formas de estructuración organizacional. Algunas ya invierten en la formación de sus empleados y en la introducción de nuevas tecnologías garantía de una mayor productividad en todos los estadios de la cadena de valor. Sin embargo, al actuar en un marco legal, político y cultural tan contrario a la cooperación en el comportamiento económico, los directivos norteamericanos solo han puesto en marcha estas reformas de manera paulatina y moderada.

El caso es distinto en el capitalismo europeo que tiene incorporado el "hábito de cooperación" entre las grandes empresas y sus gobiernos nacionales desde antes de la Segunda Guerra Mundial. No obstante, el florecimiento de la colaboración entre empresas a través de redes de productores es, realmente, algo mucho más reciente.

La forma en que pueden vincularse los grandes clientes con sus proveedores difiere de lo descripto anteriormente cuando las grandes plantas industriales operan con procesos continuos y elaboran commodities. Este tramado de relaciones inter-firma está escasamente analizado, ya que la mayor parte de la literatura ha centrado su atención en la forma que adquieren estas vinculaciones entre plantas ensambladoras y sus proveedores pymes. Burachik (1999) ${ }^{1}$ en su estudio encontró que en este caso las grandes plantas tercerizan actividades de carácter complementario, tales como tareas de mantenimiento, reparación, ejecución de obras para mejoras y ampliaciones, y otros servicios auxiliares, que no necesitan de la construcción de conocimientos y otros activos específicos. Los proveedores sólo conocen superficialmente los principios tecnológicos del proceso productivo de sus clientes. Asimismo, la forma en que las grandes plantas seleccionan a sus proveedores está gobernada

\footnotetext{
${ }^{1}$ El trabajo realizado por Burachick "Plantas grandes de proceso continuo e inserción regional; naturaleza de las demandas derivadas a pequeños proveedores manufactureros locales” analiza en una primera parte las características tecnológicas y económicas de las relaciones que se establecen entre la decena de grandes plantas radicadas en Bahía Blanca y los proveedores manufactureros locales. La segunda parte del trabajo estudia el impacto de las transformaciones experimentadas por la economía argentina desde principios de los noventa sobre la intensidad y el contenido del flujo de dichas transacciones.
} 
principalmente por condiciones de costos para un nivel mínimo de calidad dado. Licitan las obras y estimulan la competencia de precios entre proveedores rivales, no obstante, en algunas transacciones operan efectos de reputación que favorece la recontratación de proveedores ya existentes. En general, los rasgos descriptos que caracterizan al vínculo, parecen no beneficiar la aparición de elementos de cooperación.

\section{La relación proveedor-cliente de las pequeñas y medianas firmas bahienses}

La apertura económica iniciada en 1976 junto al régimen sectorial de promoción industrial instalado en la década del '80 incentivó el reemplazo de las producciones de bienes derivados de procesos productivos separables (predominantemente metalmecánica), por aquellas de insumos de uso difundido (celulosa, papel, acero, productos petroquímicos, etc.) caracterizadas por procesos productivos continuos, rígidos, intensivos en capital y en recursos naturales, que requieren una relativamente baja calificación de la mano de obra. Este cambio estructural en la industria argentina fue liderado por las grandes empresas que, generando mínimos encadenamientos productivos (desalentados por los altos índices de inflación y la caída en el nivel de actividad económica), aumentaron la brecha de productividad respecto a las pymes (Donato, V. ,1996).

En los años noventa se observa una profundización en el proceso iniciado en la década anterior referido a la expansión de las industrias procesadoras de materias primas y recursos naturales. Bajo este proceso Bahía Blanca, con las ventajas locacionales ofrecidas por su puerto y por la disponibilidad de recursos naturales, se presentó como un centro geográfico atractivo para el asentamiento de nuevas plantas grandes y la ampliación del polo petroquímico existente ${ }^{2}$.

\subsection{Caracterización de la oferta de los proveedores locales a las grandes plantas}

Desde el inicio de los '90, el proceso de privatizaciones, desregulaciones y traspaso de propiedad modificó el ambiente en el que operan las firmas proveedoras de grandes plantas. Durante este período se produjo, por un lado, un incremento de las demandas derivadas a pequeñas firmas locales debido a la mayor externalización de tareas y, por otro, una liberalización de recursos humanos calificados producto del proceso racionalizador de las grandes empresas, lo que indujo a la aparición de nuevos establecimientos pequeños y medianos.

En esta sección se analiza la información relevada en el año 2002 para una muestra de ciento once pequeñas y medianas firmas proveedoras de grandes plantas localizadas en Bahía Blanca. Una caracterización inicial de la muestra permite distinguir siete grupos de empresas según el tipo de actividad desarrollada (STA):

(1) Fabricación de piezas, componentes y repuestos: abarca, por un lado, un conjunto de proveedores centrales que fabrican e instalan por ejemplo, cañerías para transporte de fluidos o bienes para logística interna como son los pallets y bins y, por otro, empresas metalúrgicas que realizan trabajos menores como la producción e instalación de estructuras metálicas y accesorios para obras.

(2) Montaje y obras: incluye una decena de empresas que realizan el montaje industrial (ya sea civil, eléctrico, mecánico o electromecánico y combinados) asociado a las mejoras y ampliaciones

\footnotetext{
${ }^{2}$ Desde sus orígenes, la ciudad de Bahía Blanca fue núcleo de concentración y comercialización de los productos agropecuarios provenientes de la región, consolidándose un importante sector comercial y de servicios. El establecimiento de las firmas industriales giró en torno al complejo agropecuario y las actividades metalmecánicas. En los '70 se acentúa la tendencia a especializarse en la producción de alimentos (notable auge de la industria aceitera) y se proyecta la instalación del Complejo Petroquímico. Durante la década del '80, se pone en marcha dicho Complejo (iniciada la construcción en la década anterior) y la Usina Termoeléctrica (Esandi, 1996). Mientras que en la década del '90 se crea EG3 Asfalto como una subsidiaria de EG3 S.A. para incrementar el valor agregado del asfalto que se obtenía del proceso de refinación. Se instala la empresa agroindustrial multinacional Cargill, se amplia la capacidad productiva del Polo Petroquímico y se radica la productora de fertilizantes Profertil.
} 
de las grandes plantas existentes y a la radicación de las nuevas. El resto de este grupo, de menor importancia relativa como proveedores de este tipo de plantas, comprende a firmas que realizan obras viales. instalación de redes de gas, impermeabilización $\mathrm{v}$ aislamiento térmico como tareas auxiliares que completan las obras.

(3) Mantenimiento y reparación: contiene aquellas empresas que realizan tareas rutinarias de mantenimiento preventivo de equipos electrógenos, cañerías, limpiezas hidráulicas, arenado y pintura, y reparación de equipos mecánicos, equipos electrónicos, etc. Las tareas de mantenimiento realizadas por estas empresas también se encuentran asociadas a las paradas de planta efectuadas anualmente por las grandes firmas.

(4) Logística: se incluye a un pequeño grupo de empresas que prestan servicios asociados a necesidades referidas a la carga y descarga para el almacenaje y el despacho de productos terminados y también a firmas que realizan tratamiento de los residuos industriales.

(5) Servicios técnicos: comprende un número reducido de empresas que ofrecen servicios técnicos de telecomunicaciones, diseño de ingeniería, mecánica y asistencia técnica de transmisiones mecánicas. Parte de estas firmas poseen mano de obra especializada que es contratada por otros establecimientos locales.

(6) Servicios auxiliares: este grupo comprende, entre las más importantes a las firmas que brindan servicios tales como vigilancia, limpieza, servicio de comedor, transporte de personal, parquizado y servicio de control de plagas.

(7) Alquiler, comercialización, distribución: estas empresas tienen como actividad principal el alquiler, la comercialización y distribución de productos fabricados por otras firmas (materiales eléctricos, equipos de control automatizados) y como actividad secundaria la fabricación (tableros eléctricos, mangueras hidráulicas, etc.) y la prestación de servicios.

El porcentaje de empresa distribuídas en grupos de actividad se presenta en el cuadro 1. El mismo permite observar que la oferta de los proveedores se caracteriza sustancialmente por la prestación de servicios que desarrollan actividades auxiliares con distinto grado de complementariedad.

Cuadro 1: Pequeñas y medianas empresas según tipo de actividad (STA)

\begin{tabular}{cc}
\hline $\begin{array}{c}\text { Empresas según tipo de } \\
\text { actividad }\end{array}$ & Empresas encuestadas \\
& $\begin{array}{c}\text { Porcentajes de } \\
\text { empresas por grandes } \\
\text { rubros }\end{array}$ \\
\hline
\end{tabular}

1. Fabricación de piezas, componentes y repuestos

2. Montaje y obras

$20-16.1$

3.Mantenimiento y

reparación

4. Logística

5. Servicios técnicos

10

8.1

6. Servicios auxiliares

7. Alquiler,

118.9

comercialización, distribución

Las firmas también pueden agruparse según edad y tamaño. Esta distribución intenta visualizar su sendero madurativo utilizando datos de corte transversal. Se estableció un rango de edades para identificar firmas nuevas (menores a 2 años), adolescentes (entre 3 y 6 años), jóvenes (entre 7 y 12 años ), maduras (entre 13 y 25 años) y tradicionales (más de 25 años) (Figura 1) 
(Leonardi, V. y Viego, V., 1999). Por otro lado, para definir el tamaño de las empresas se adoptó la facturación anual ${ }^{3}$ realizando la siguiente distinción: microempresa: menos de \$ 50.000; pequeña: de \$ 50.000 a \$ 160.000; intermedia: \$160.000 a \$1.000.000; mediana \$1.000.000 a \$ 3.000.000; grande: más de 3.000 .000

El análisis de la distribución de las firmas según edad pone de relieve dos cuestiones. Por un lado, la mayor proporción de las firmas son maduras, lo cual refleja la capacidad de este grupo de empresas para adaptarse a condiciones cambiantes del ambiente. Por otro lado, también es relevante la proporción de firmas jóvenes (entre 7 y 12 años) resultado fundamentalmente de la liberalización de recursos humanos calificados anteriormente comentado ${ }^{4}$. Asimismo, se observa que no existen firmas nuevas en el grupo de empresas encuestadas.

La Figura 2 revela que el $46 \%$ de las empresas proveedoras locales tienen un tamaño intermedio entre la pequeña y la mediana empresa, las cuales en conjunto representan el segundo estrato de importancia (41\%).

Figura 1: Distribución de las firmas según edad (\%)

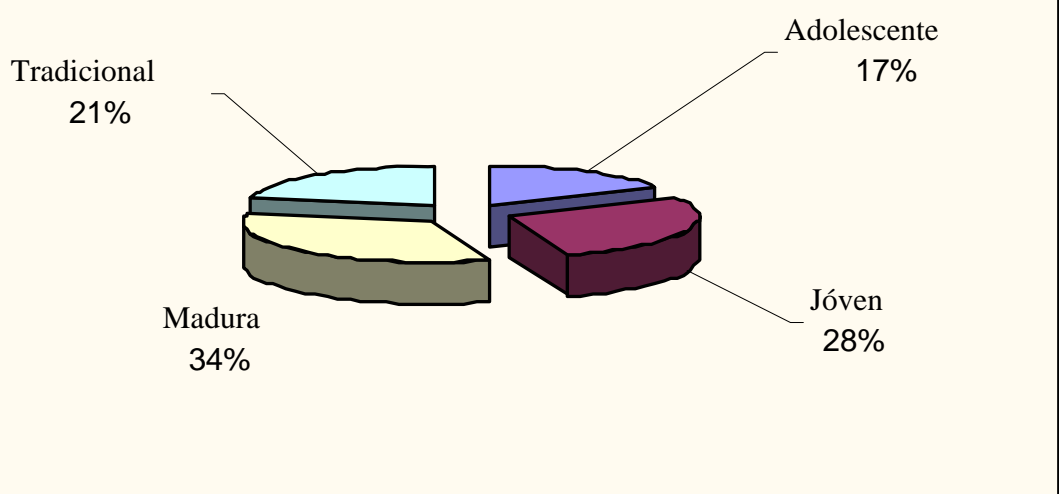

Fuente: Elaboración propia sobre la base de datos de la encuesta 2002. Dpto. de Economía. U.N.S.

Figura 2: Tamaño de las empresas según facturación anual (\%)

\footnotetext{
${ }^{3}$ Como criterio asimilable al utilizado por los Censos Nacionales Industriales 1985-1994. Según ellos la clasificación de acuerdo al valor de producción distingue: microempresas: menos de \$50.000; pequeña: de \$50.000 a 140.000; intermedia: de $\$ 140.000$ a 700.000 y mediana: de $\$ 700.000$ a 3,5 millones.

${ }^{4}$ La antigüedad promedio del conjunto de firmas es de 16 años, apareciendo como casos extremos el sector de fabricación, con una antigüedad promedio de 26 años y el sector que suministra servicios técnicos como el nobel, con 9 años de edad promedio.
} 


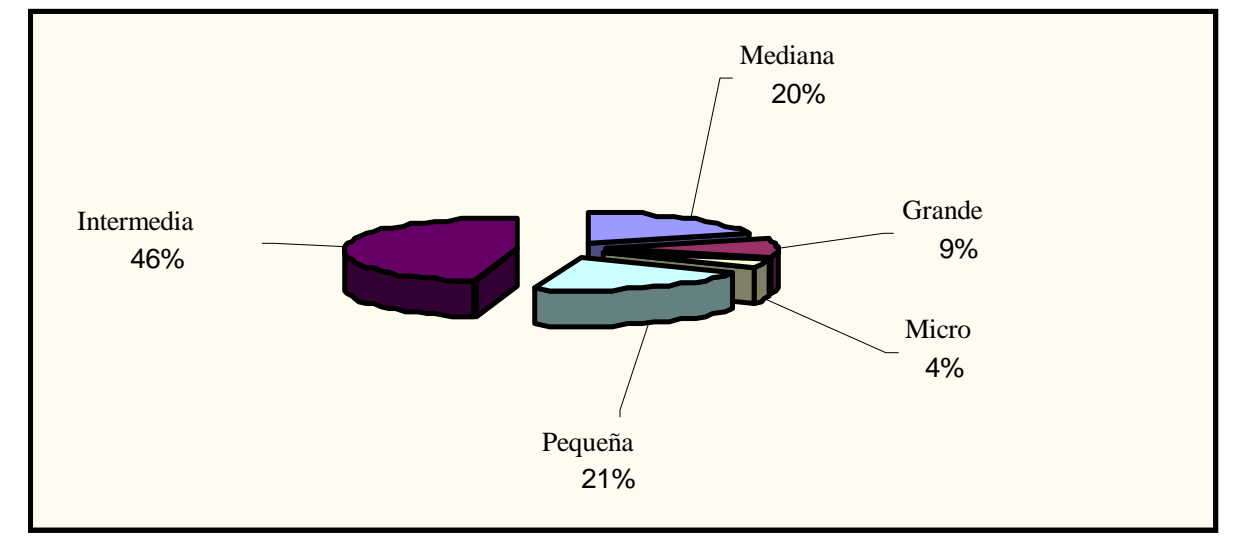

Fuente: Elaboración propia sobre la base de datos de la encuesta 2002. Dpto. de Economía. U.N.S.

Algunas otras características del grupo de empresas encuestas son:

La edad promedio de los socios principales es de 47 años, los que tienen estudios universitarios completos en el $55 \%$ de los casos.

El 80\% de las empresas realizaron inversiones en el período 1997/2001. En el 30\% de los casos se invirtió para proveer a un gran cliente.

La incorporación de mejoras y de nuevas tecnologías se destinaron al sector de distribución (30\%), fabricación (27\%) y comercialización (22\%).

La actividad productiva que absorbe el mayor número de personal ocupado (permanente y contratado) en el año 2001 es la de servicios auxiliares (31\%), seguida de montaje y obras (18\%) y mantenimiento y reparación (17\%).

2.2 Principales características del vínculo entre las grandes plantas y los pequeños y medianos proveedores locales.

Es importante analizar el nivel de concentración de las ventas a los clientes grandes por su relevancia como indicador para medir el grado de dependencia de los pequeños y medianos proveedores locales. La figura 3 muestra el nivel de significatividad de la demanda de bienes y servicios para cada sector, por parte de las grandes empresas industriales. Como se observa el mayor impacto de la misma recae básicamente en las firmas que realizan montaje y obras, mantenimiento y reparaciones y fabricación de piezas, componentes y repuestos. El resto de los grupos de empresas diversifican más sus ventas entre otros pocos grandes y numerosos pequeños clientes del sector privado no exclusivamente bahiense, siendo casi inexistente la presencia del sector público en este aspecto.

Figura 3: Participación de las grandes empresas en la facturación media anual de los pequeños proveedores (\%)

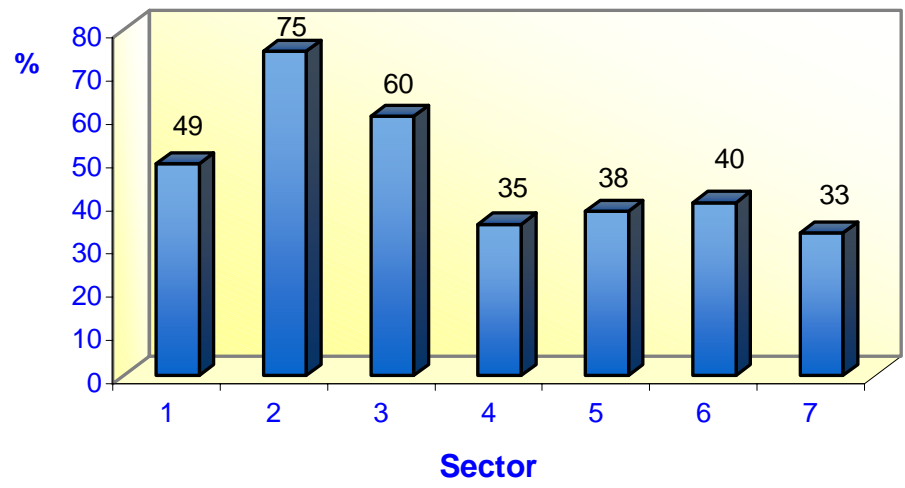


Fuente: Elaboración propia sobre la base de datos de la encuesta 2002. Dpto. de Economía. U.N.S. *Sectores: Clasificación de las empresas según el tipo de actividad que desarrollan: (1)Fabricación de piezas, componentes y repuestos; (2) Montaje y obras; (3) Mantenimiento y reparación; (4) Logística; (5) Servicios técnicos; (6) Servicios auxiliares; (7) Alquiler, comercialización, distribución.

Respecto a la especificidad de los activos involucrados en las transacciones grandes-pymes, según los resultados obtenidos en las encuestas, se observa que los pequeños y medianos proveedores manufactureros, en general, no conocen la tecnología básica de producción de las grandes plantas, a lo cual se le suma la baja especificidad de los activos involucrados en las transacciones. Únicamente el caso de las firmas que ofrecen servicios técnicos parecen poseer un mayor caudal de conocimiento y de activos específicos. En resumen, las actividades complementarias externalizadas por las grandes firmas al no requerir la construcción de conocimientos u otros activos específicos conduce a un alto grado de sustituibilidad entre proveedores.

Por otro lado, el análisis de las formas de contratación indica que las grandes plantas al momento de contratar a sus proveedores lo hace a través del contrato directo en el 38\% de los casos. Le sigue en orden de importancia el concurso de precios (32\%) y la subcontratación (15\%). La naturaleza y escala del pedido solicitado por las grandes plantas industriales a sus proveedores, determina la duración de las relaciones contractuales. El período predominante de duración de los contratos es un año o menos de un año (64\% de los casos) sobresaliendo, en segundo lugar, la duración según obra, trabajo, servicio o compra (25\%).

Estos elementos indican que las vinculaciones entre las grandes plantas industriales bahienses y los pequeños proveedores manufactureros no son estables. La baja especificidad de los activos y la variabilidad en la frecuencia de las transacciones determina que el mercado sea el principal medio de gobernancia de las vinculaciones inter-firma, impidiendo el surgimiento de contratos de largo plazo que alienten alguna forma de cooperación.

\section{Análisis econométrico, método de estimación e interpretación de resultados.}

En esta sección se intenta analizar la importancia que reviste para una pequeña y mediana empresa ser proveedor de una planta grande que manufactura commodities. La variable endógena considerada es el desempeño de la empresa, medido a través de la evolución de la facturación. La misma se define como una variable dicotómica que toma el valor 1 cuando ha aumentado la facturación en el período 1997-2001, y cero si no ha aumentado ${ }^{5}$. Dado que la variable dependiente es una variable dicotómica cualitativa el modelo econométrico empleado para realizar la estimación es un probit ${ }^{6}$. La estimación del modelo probit aquí utilizado se formalizó mediante el método de máxima verosimilitud a través del programa stata.

El modelo econométrico parte de una función que incorpora a tres grupos de variables y a la facturación de las grandes plantas como explicativas del desempeño:

(a) X1: Características de la empresa

(b) X2: Inversión

(c) X3: Facturación a las grandes plantas

(d) X4: Tipo de actividad productiva

\footnotetext{
${ }^{5}$ También podría haberse utilizado como indicador de eficiencia el aumento de los activos físicos, aumento en el nivel de empleo o alguna combinación de los tres indicadores (Storey, 1994). El empleo no se consideró dado que la mayor parte de las firmas realizan servicios a la producción con un número significativo de personal transitorio que oscila según el trabajo requerido. Por otro lado, el indicador de niveles de activos físicos no pudo emplearse debido a la falta de información.

${ }^{6}$ Para explicar el comportamiento de una variable dependiente dicotómica es preciso utilizar una función de distribución probabilística acumulada. El modelo de estimación que surge de una distribución acumulativa normal se conoce como modelo probit.
} 
Por lo tanto, el modelo queda determinado por:

$$
Y 1=\alpha+\beta X 1+\delta X 2+\phi X 3+\lambda X 4+\mu
$$

El primer grupo de variables presenta las características de la empresa: tamaño (X11) y antigüedad de la firma (X12). El tamaño de la firma se definió a través del nivel de facturación y la variable toma los siguientes valores: 1: microempresa, 2: pequeña, 3: intermedia, 4: mediana y 5: grande. Se espera que a mayor tamaño mejor sea el desempeño de la empresa. Esto puede fundamentarse en que la dimensión está fuertemente asociado a las debilidades organizacionales y a los factores que determinan la capacidad tecnológica de las firmas (Puppo, 2003). Respecto a la edad de la firma, se espera que a mayor edad menor sea la probabilidad de que una pyme obtenga un desempeño positivo. Esto se basa en que los propietarios de las empresas de menor edad, en general, son más jóvenes y tienen mayor nivel educativo, lo que en general se traduce en un mayor dinamismo.

El segundo grupo considera si la empresa ha invertido o incorporado tecnología. Este grupo incluye dos variables dummies: X21 toma el valor 1 si la firma realizó inversiones y cero en el resto de los casos, mientras que X22 toma el valor 1 si la empresa ha incorporado tecnología en el proceso de producción y cero en el resto. En ambos casos se espera que exista una relación positiva entre estas dos variables explicativas y la variable endógena. Este grupo de variables fue incluido en el análisis dado que la inversión es considerada una variable estratégica que influye sobre el desempeño ${ }^{7}$.

A fin de captar los efectos de que la vinculación con las grandes plantas tiene sobre el desempeño de una firma se tomó la variable X3 la cual representa el porcentaje de facturación de las pymes hacia las grandes empresas. El signo esperado de esta variable es positivo, lo cual estaría indicando que a medida que aumenta el monto de facturación a las grandes plantas crece la probabilidad de obtener un desempeño positivo.

Finalmente, el cuarto grupo representa la actividad productiva de las empresas. Como se mencionó anteriormente las firmas están agrupadas por tipo de actividad en siete categorías. A fin de disminuir el grado de complejidad de análisis, se emplearon cinco variables dummies tomando como categoría base a la fabricación de piezas y componentes. En este grupo las variables consideradas son las siguientes: X41: toma el valor 1 si la empresa realiza actividades de montaje y obras y cero en el resto, X42: toma el valor 1 si la empresa realiza actividades de mantenimiento y reparación y cero en el resto, X43: toma el valor si la empresa realiza actividades de logística y cero en el resto, X44: toma el valor 1 si la empresa realiza actividades de servicios técnicos y cero en el resto, X45: toma el valor 1 si la empresa realiza actividades de distribución, alquiler, comercialización y servicios auxiliares y cero en el resto. Esta caracterización servirá para determinar qué tipo de actividad tiene o no probabilidad de impactar positivamente en el desempeño en relación a la actividad de fabricación y de esta forma poder realizar comparaciones.

\footnotetext{
${ }^{7}$ La teoría tradicional de la inversión sugiere que el rendimiento de la empresa en un área de negocio es proporcional al tamaño de la inversión (Ansoff, 1990).Así, existe una masa crítica mínima que debe alcanzar la inversión para que la misma sea rentable. Esto implica que el nivel de inversión realizado por cada firma tendrá un impacto positivo sobre el nivel de ganancias, siempre que el monto de inversión supere la masa crítica, ingresando de este modo en el conjunto de variables de decisión estratégica. También existe un nivel de masa óptima que representa el nivel de inversión más allá del cual el rendimiento comienza a declinar debido a la disminución de la velocidad de respuesta de la organización y al desarrollo burocrático de la empresa.

Siguiendo este argumento, puede definirse una nueva dimensión en la explicación de la conducta de la firma individual, denominada "inversión estratégica", entendida como el compromiso de recursos que una empresa decide destinar a un determinado curso de acción. La inversión estratégica, por lo tanto, incluye no sólo el monto destinado a la adquisición de equipos e instalaciones, sino también al desarrollo del producto y del mercado, así como a la asistencia a la gerencia, producción, ventas, etc.
} 
Los resultados del ajuste se realizaron en principio para el conjunto de las variables (Cuadro 2) y luego para cada uno de los cuatro grupos de variables (Cuadro 3).

Cuadro 2: Estimación para el conjunto de variables

Número de obs. $=111 ; \quad$ LR chi2 $(10)=22.59 ; \quad$ Prob $>$ chi2 $=0.0123$

\begin{tabular}{|c|c|c|c|c|c|c|}
\hline y1 & Coef. & Std. Err. & $\mathrm{z}$ & $\mathrm{P}>|\mathrm{z}|$ & \multicolumn{2}{|c|}{ [95\% Conf. Interval] } \\
\hline x11 & .1767368 & .1640232 & 1.08 & 0.281 & -.1447427 & .4982164 \\
\hline$x 12$ & -.0138956 & .0100217 & -1.39 & 0.166 & -.0335378 & .0057465 \\
\hline$x 21$ & 1.149793 & .4794192 & 2.40 & 0.016 & .2101483 & 2.089437 \\
\hline$x 22$ & -.0006569 & .3327086 & -0.00 & 0.998 & -.6527537 & .6514399 \\
\hline$x 3$ & .0061854 & .0040539 & 1.53 & 0.127 & -.0017601 & .014131 \\
\hline x41 & .4193852 & .4696834 & 0.89 & 0.372 & -.5011774 & 1.339948 \\
\hline$x 42$ & -.4787982 & .5592319 & -0.86 & 0.392 & -1.574873 & .6172761 \\
\hline$x 43$ & .4723486 & .5162542 & 0.91 & 0.360 & -.5394911 & 1.484188 \\
\hline 44 & -.1790932 & .4832513 & -0.37 & 0.711 & -1.126248 & .768062 \\
\hline$x 45$ & .3405064 & 4190755 & 0.81 & 0.416 & -.4808665 & 1.161879 \\
\hline cons & -2.235801 & .6997573 & -3.20 & 0.001 & -3.6073 & -.8643017 \\
\hline
\end{tabular}

Fuente: Elaboración propia sobre la base de datos de la encuesta 2002. Dpto. de Economía. UNS.

De la observación del cuadro 2 pueden extraerse algunas conclusiones acerca de la bondad del ajuste efectuado.

El estadístico LR muestra como significativo al conjunto de variables elegidas para explicar la probabilidad de que una firma tenga un desempeño positivo, el valor computado de este estadístico es de 22.59, con una probabilidad asociada de 0.0123; esto permite rechazar la hipótesis conjunta de que todos los coeficientes de las variables son cero con un alto nivel de confianza. Sobre la base del estadístico z se observa que la única variable que se manifiesta como significativa para explicar la probabilidad de que una firma tenga un desempeño positivo es la inversión (X21) a un nivel de significatividad del 2 por ciento.

Por otro lado, examinando los resultados que presenta el cuadro 3 (ver anexo regresiones 1 ; 2; 3 y 4), puede apreciarse que según el estadístico z las variables que se muestran como significativas en la determinación del desempeño de la firma son las relacionadas con las características de la firma (X11 y X12), la inversión realizada (X21) y la facturación a las grandes plantas (X3). La incorporación de tecnología (X22) no se manifiesta como significativa e incluso muestra un signo opuesto al esperado en la regresión 1, lo cual parece deberse a un problema de multicolinealidad entre las variables.

Cuadro 3: Estimaciones por grupo de variables

\begin{tabular}{|c|c|c|c|c|c|c|}
\hline $\begin{array}{c}\text { Grupo de } \\
\text { variables } \\
\text { independientes }\end{array}$ & $\operatorname{Reg}$ & ión 1 & \multicolumn{2}{|c|}{ Regresión 2} & Regresión 3 & Regresión 4 \\
\hline \multirow{2}{*}{$\begin{array}{c}\text { Características de } \\
\text { la empresa }\end{array}$} & $X 11$ & $X 12$ & & & & \\
\hline & $\begin{array}{c}2.57 \\
(0.010)\end{array}$ & $\begin{array}{c}-1.80 \\
(0.072)\end{array}$ & & & & \\
\hline \multirow{2}{*}{$\begin{array}{c}\text { Inversión e } \\
\text { incorporación de } \\
\text { tecnología }\end{array}$} & & & $x 21$ & $x 22$ & & \\
\hline & & & $\begin{array}{c}2.39 \\
(0.017)\end{array}$ & $\begin{array}{c}0.67 \\
(0.502)\end{array}$ & & \\
\hline
\end{tabular}




\begin{tabular}{|c|c|c|c|c|c|c|c|c|}
\hline \multirow{2}{*}{$\begin{array}{c}\text { Tipo de actividad } \\
\text { productiva }\end{array}$} & & & $X 41$ & $X 42$ & $X 43$ & $X 44$ & $X 45$ & \\
\hline & & & $\begin{array}{c}1.50 \\
(0.133)\end{array}$ & $\begin{array}{c}-0.56 \\
(0.579)\end{array}$ & $\begin{array}{c}1.35 \\
(0.177)\end{array}$ & $\begin{array}{c}0.30 \\
(0.764)\end{array}$ & $\begin{array}{c}0.79 \\
(0.429)\end{array}$ & \\
\hline \multirow{2}{*}{$\begin{array}{l}\text { Vinculación con } \\
\text { las grandes } \\
\text { plantas }\end{array}$} & & & & & & & & $x 3$ \\
\hline & & & & & & & & $\begin{array}{c}1.66 \\
(0.097)\end{array}$ \\
\hline$L R$ & $\begin{array}{c}9.04 \\
(0.0109)\end{array}$ & $\begin{array}{c}10.45 \\
(0.0054)\end{array}$ & & & $\begin{array}{c}5.50 \\
(0.3583)\end{array}$ & & & $\begin{array}{c}2.80 \\
(0.094)\end{array}$ \\
\hline
\end{tabular}

Fuente: Elaboración propia sobre la base de datos de la encuesta 2002. Dpto. de Economía. U.N.S.

A juzgar por las magnitudes relativas de sus respectivos coeficientes y probabilidades asociadas podría decirse que la importancia relativa de la inversión (X21) y del tamaño de la firma (X11) es superior al resto.

Los coeficientes de las variables explicativas presentan los signos esperados. Así se observa que: las variables X11, X21 y X3 están relacionadas positivamente con la variable endógena, mientras que X12 se relaciona negativamente. Por un lado, la relación positiva entre X11, X21 y X3 con Y1 indica que a medida que aumenta el tamaño de la firma, la firma invierte y a medida que aumenta el nivel de facturación a las grandes plantas, se incrementa la probabilidad de que una pequeña y mediana firma tenga un desempeño positivo. Por otro lado, la relación negativa entre X12 e Y1 indica que cuando aumenta la edad de la firma la probabilidad de que la empresa tenga un desempeño favorable disminuye. Esto puede explicarse considerando que una de las características que distingue a las pymes es su orientación estrictamente personal. Es decir, la conjunción de las funciones de gestión, administración y control en el dueño imprime un rasgo peculiar dando lugar a una fuerte asimilación de las características personales del individuo. Según lo observado en las encuestas parecen ser más dinámicos los propietarios de las empresas más nuevas. En general, los propietarios de las empresas más antiguas poseen menor nivel de estudios formales y son más conservadores, es decir, son más renuentes al riesgo, lo cual parece jugar desfavorablemente en el desempeño.

Asimismo, el estadístico z parece indicar que el tipo de actividad desarrollado por las firmas proveedoras no resulta significativo para explicar la probabilidad que la empresa tenga un desempeño positivo. El estadístico LR (análogo al estadístico $\mathrm{F}$ en las regresiones lineales) muestra que el grupo de variables relacionadas con el tipo de actividad desarrollado por la empresa no es importante para explicar el comportamiento de la variable endógena.

Comparando los resultados presentados en los cuadro 2 y 3 puede observarse que en la regresión donde se incluyen todas las variables explicativas X11, X12 y X3 dejan de ser significativas lo cual puede atribuirse a un problema de multicolinealidad. Así, por ejemplo, la variable tamaño de la firma manifiesta tener un mayor grado de significatividad cuando se realiza la regresión sólo para el primer grupo de variables, pero pierde significatividad al incorporar la inversión, esto señala que existe una relación positiva entre tamaño e inversión. Es decir, la probabilidad de que una firma invierta depende de su tamaño (ver anexo regresión 5).

En función de los resultados obtenidos se puede plantear un nuevo modelo para explicar la probabilidad de que una firma tenga un buen desempeño. Teniendo en cuenta a las variables que muestran ser significativas puede excluirse del análisis el grupo de variables que consideran el tipo de actividad desarrollada y la variable X22 que refleja la incorporación de tecnología en el proceso productivo ya que la misma revela no ser significativa dentro del grupo de variables (debido a problemas de multicolinelidad) como tampoco muestra mejorar la estimación si es incorporada al modelo (ver anexo, regresión 6).

El modelo final considerado para explicar el desempeño de una empresa es el siguiente: 
Donde:

$$
Y 1=\alpha+\beta 1 \times 11+\beta 2 \times 12+\delta 1 \times 21+\phi X 3+\mu
$$

X11: tamaño de la firma

$\mathrm{X} 12$ : edad de la firma

X21: inversión

X3: nivel de facturación a las grandes plantas.

La estimación realizada para este modelo se presenta en el cuadro 4

$$
\text { Cuadro 4: Estimación del modelo final }
$$

Número de obs. = 111; LR chi2(4) $=17.59 ; \quad$ Prob $>$ chi2 $=0.0015$

\begin{tabular}{ccccccc}
\hline $\mathrm{y} 1$ & Coef. & Std. Err. & $\mathrm{z}$ & $\mathrm{P}>|\mathrm{z}|$ & \multicolumn{2}{c}{ [95\% Conf. Interval] } \\
\hline $\mathrm{x} 11$ & .2644948 & .1493598 & 1.77 & 0.077 & -.028245 & .5572346 \\
$\mathrm{x} 12$ & -.0140618 & .0092475 & -1.52 & 0.128 & -.0321866 & .0040629 \\
$\mathrm{x} 21$ & .967698 & .4274522 & 2.26 & 0.024 & .1299072 & 1.805489 \\
$\mathrm{x} 3$ & .0059931 & .0038307 & 1.56 & 0.118 & -.001515 & .0135012 \\
cons & -2.189516 & .6308779 & -3.47 & 0.001 & -3.426014 & -.9530178 \\
\hline
\end{tabular}

Fuente: Elaboración propia sobre la base de datos de la encuesta 2002. Dpto. De Economía. UNS.

El estadístico LR muestra como significativo al conjunto de variables elegidas para explicar la probabilidad de que una firma tenga un desempeño positivo. El valor computado de este estadístico es de 17.59, menor al modelo original mientras que la probabilidad asociada es en este caso mayor (0.0015).

En esta estimación, en base al estadístico z, puede afirmarse que las únicas variables que se manifiestan como significativa son el tamaño de la firma (X11) y la inversión (X21). Sin embargo, si se compara este resultado con el del cuadro 3 puede observarse que las variables X11 pierden significatividad, mientras que las variables X12 y X3 dejan de ser significativas lo cual, como se mencionó anteriormente puede atribuirse a un problema de multicolinealidad entre las variables.

Dado que en este tipo de modelos los coeficientes no pueden interpretarse directamente, se realizó una nueva estimación a fin de observar los efectos de un cambio marginal en las variables explicativas sobre la variable endógena, siempre medida para el período 1997-2001 (cuadro 5) .

Cuadro 5: Análisis de los cambios marginales

\begin{tabular}{|c|}
\hline $\mathrm{dF} / \mathrm{dx}$ \\
\hline
\end{tabular}

Número de obs. = 111; LR chi2(4) = 17.59; Prob > chi2 = 0.0015

$\begin{array}{rrlccccc}\text { x11 } & .0929487 & .0521011 & 1.77 & 0.077 & 2.99099 & -.009168 & .195065 \\ \times 12 & -.0049416 & .0032343 & -1.52 & 0.128 & 17.4324 & -.011281 & .001398 \\ \times 21^{*} & .2789978 & .0907725 & 2.26 & 0.024 & .792793 & .101087 & .456909 \\ \times 3 & .0021061 & .0013399 & 1.56 & 0.118 & 62.1892 & -.00052 & .0047\end{array}$

Fuente: Elaboración propia sobre la base de datos de la encuesta 2002. Dpto. de Economía. U.N.S.

La estimación pone de manifiesto que la variable que muestra un mayor impacto sobre el cambio en la probabilidad de obtener un desempeño positivo es la inversión. De este modo, cuando las 
pequeñas y medianas firmas deciden invertir, su probabilidad de obtener un resultado positivo aumenta en un 28 por ciento. ${ }^{8}$

Por otra parte, es importante recordar que estos modelos son muy sensibles a errores de especificación. Podría considerarse que si bien las variables tenidas en cuenta permiten abarcar un espectro bastante amplio de los aspectos determinantes del desempeño de la firma, existe otro grupo de variables principalmente relacionadas con las características de los empresarios que podrían tener un impacto significativo sobre la variable dependiente, lo cual podría incluirse en futuras investigaciones.

\section{Reflexiones finales}

Dado que en la última década se han expandido las actividades intensivas en recursos naturales y el procesamiento de commodities en grandes plantas, resulta interesante analizar la posible formación de redes de empresas o encadenamientos productivos entre ellas y las pymes locales, como una forma de incorporar el aprendizaje tecnológico y de comercialización que ayude a mejorar las condiciones de competitividad de estas últimas.

En los escasos trabajos realizados en torno a este tema, se ha encontrado que los principales impactos inter-industriales se vinculan con la demanda por parte de las grandes firmas de insumos y de servicios, que se derivan a las pequeñas y medianas empresas, tales como tareas de mantenimiento y reparación, montaje y obras, servicios técnicos, logística, tratamiento de residuos, comercialización y distribución todas estas actividades resultan auxiliares con distinto grado de complementariedad al proceso productivo. De allí que la formación de redes sea nula y los encadenamientos productivos, si bien con sustancial grado de eslabonamiento hacia atrás, son escasos y débiles hacia adelante.

Esta derivación principalmente de "servicios industriales" conduce al desarrollo de tareas con muy poca especificidad en sus activos tanto físicos como de conocimientos, lo cual hace a las transacciones altamente vulnerables, dada la facilidad de sustitución entre proveedores. Así, las características del vínculo generado en este caso entre proveedor-cliente permite concluir que, en general, no existe un impacto significativo sobre el desempeño de una pyme local. Este resultado es corroborado por el análisis econométrico realizado. Del mismo se infiere que la inversión se convierte en la variable exógena que posee mayor importancia al explicar la probabilidad de que una empresa tenga un desempeño positivo.

Si bien los rasgos que adquieren las relaciones inter-firma de las industrias de procesos continuos que elaboran commodities no son propicios para crear un ambiente fructífero para la cooperación, cabe destacar la debilidad generalizada a nivel nacional para modificar las pautas históricas de interrelación de firmas.

Actualmente, en Bahía Blanca, la inquietud de actores sociales tales como el municipio, la asociación empresaria APYME y la buena predisposición del área gerencial de las grandes plantas ha promovido reuniones informales en las que se han analizado posibles extensiones de los contratos, aceptación de uniones transitorias de pequeños contratistas por parte de las grandes empresas para proveerlas y la intensificación de los programas que ayuden a las pymes locales en su proceso de

\footnotetext{
${ }^{8}$ Esta conclusión coincide con resultados hallados en una investigación realizada en el año 1999 para pymes localizadas en Bahía Blanca correspondientes a distintos sectores industriales (Leonardi, Viego, 1999). En dicha investigación se encontró que las firmas que realizaron inversiones sustancialmente mayores a las restantes y que fueron canalizadas hacia nuevas tecnologías o bienes de capital tuvieron un desempeño positivo. El análisis econométrico realizado en este trabajo permite corroborar este resultado, si bien, aquí sólo se considera si la empresa ha invertido o no sin tener en cuenta el monto de inversión realizado y el destino de la inversión.
} 
reconversión. Pero todo ello no es más que un paliativo frente a la falta de desarrollo de una estrategia pyme sustentable en el tiempo que integre organismos representativos de la sociedad civil (cámaras empresarias, agrupaciones del sector, entes de capacitación formal e informal, etc.) y del Estado en sus distintos niveles de representación.

\section{BIBLIOGRAFÍA}

Ansoff, H. y McDonell, E., (1997) La dirección estratégica en la práctica empresarial, Ed. Addison - Wesley Iberoamericana, Estados Unidos.

Bourgault, M., Lefebvre, L y Lefebvre, E., (1995). "Critical capabilities and performance of small Subcontracting firms in the aerospace industry”, Productivity and Quality Management Frontiers, Vol. V, Georgia, Industrial Engineering and Management Press,.

Burachik, G., (1999) "Relación cliente-proveedor local: las plantas de proceso continuo en Argentina”, Comercio Exterior, Vol 49, Nro 12, dic., México.

DeBresson, Ch. y Amesse, F., (1991) "Networks of Innivators: A Rewiew”, Research Policy, Nro.20.

De Toni, A., Nassimbeni, G., Tonchia, S., (1995) "Pequeñas firmas locales dentro de la cadena de oferentes cambios y perspectivas”, Small Business Economics, Nro.7.

Donato, V., (1996) "Incertidumbre ambiental y procesos productivos de alta densidad contractual: La dinámica estructural de la industria argentina durante el período de la megainflación (1975-190)", Desarrollo Económico, vol. 35, № 140, Buenos Aires.

Esandi, J. (1996) Crecimiento y perspectivas de desarrollo regional en Bahía Blanca . Bahía Blanca de ayer a hoy. EDIUNS, Bahía Blanca,.

Gatto, F. y Yoguel, G., (1993) "Las PyMEs Argentinas en una etapa de transición productiva y tecnológica”, Kosaccof, B. y otros: El desafío de la competitividad, CEPAL/Alianza Editorial, Buenos Aires.

García Casal, I . y Leonardi, V. (1999). "Relacion proveedor - cliente: el caso de los pequeños contratistas industriales de Bahía Blanca"; Red Pyme Mercosur, Mar del Plata.

Klein, B., Crawford, R. y Alechian, A., (1994). "Integración vertical, rentas apropiables y el proceso competitivo de contratación”, La naturaleza económica de la empresa, Ed. Alianza, Madrid.

Leonardi, V. y Viego, V., (1999). “Configuraciones ambiente-estrategia-desempeño. Interpretaciones en torno a las pymes industriales Bahienses”. Red Pyme Mercosur, Mar del Plata.

Noorderhaven, N.; Nooteboom, B.; y Berger, H., (1990) "Exploring determinants of perceived interfirm dependence in industrial supplier relations”, Discussion Paper 115, Center for Economic Research, Tilburg University.

Powell, W. et al, (1990 )“Neither Market nor Hierarchy: Network Forms of Organization”, Research in Organizational Behavior (comps.) Greenwich, Connecticutt: JAI Press.

Storey, D., (1985).Understanding the small business sector, Routledge. Londres, 1994.

Williamson, O., (1989)Las instituciones económicas del capitalismo, Fondo de Cultura Económica, Méjico. 


\section{APENDICE ECONOMETRICO}

\section{Estimaciones por grupo de variables}

Regresión 1: Relación entre desempeño y características de la empresa

Número de obs. $=111$

LR chi2(2) $=9.04$

Prob $>$ chi2 $=0.0109$

Log likelihood $=-66.806619$

Pseudo R2 $=0.0634$

\begin{tabular}{ccccccc}
\hline $\mathrm{y} 1$ & Coef. & Std. Err. & $\mathrm{z}$ & $\mathrm{P}>|\mathrm{z}|$ & \multicolumn{2}{l}{ [95\% Conf. Interval] } \\
\hline $\mathrm{x} 11$ & .3444711 & .1342445 & 2.57 & 0.010 & .0813568 & .6075855 \\
$\mathrm{x} 12$ & -.0165291 & .0091889 & -1.80 & 0.072 & -.034539 & .0014807 \\
cons & -1.186405 & .4331452 & -2.74 & 0.006 & -2.035354 & -.3374559 \\
\hline
\end{tabular}

Regresión 2: Relación entre desempeño e inversión e incorporación de tecnología

Número de obs. $=111$

LR chi2(2) $=10.45$

Prob $>$ chi2 $=0.0054$

Log likelihood $=-66.103232$

Pseudo R2 $=0.0732$

\begin{tabular}{rcccccc}
\hline $\mathrm{y} 1$ & Coef. & Std. Err. & $\mathrm{z}$ & $\mathrm{P}>|\mathrm{z}|$ & [95\% Conf. Interval] \\
\hline & & & & & & \\
$\mathrm{x} 21$ & 1.023264 & .4274358 & 2.39 & 0.017 & .1855048 & 1.861022 \\
$\mathrm{x} 22$ & .201323 & .2998297 & 0.67 & 0.502 & -.3863324 & .7889784 \\
cons & -1.407105 & .3813373 & -3.69 & 0.000 & -2.154513 & -.6596977 \\
\hline
\end{tabular}

Regresión 3: Relación entre desempeño y tipo de actividad productiva de las empresas proveedoras

Número de obs. $=111$

LR chi2(5) $=5.50$

Prob $>$ chi2 $=0.3583$

Log likelihood $=-68.577849$

Pseudo R2 $=0.0385$

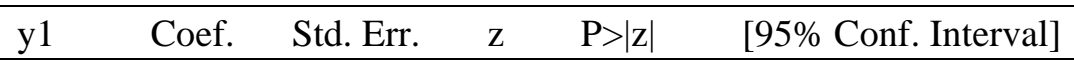




$\begin{array}{rrlrlll}\text { x41 } & .6147727 & .4095549 & 1.50 & 0.133 & -.1879402 & 1.417485 \\ \text { x42 } & -.2929318 & .5274889 & -0.56 & 0.579 & -1.326791 & .7409275 \\ \text { x43 } & .6744898 & .499918 & 1.35 & 0.177 & -.3053315 & 1.654311 \\ \text { x44 } & .1330947 & .442387 & 0.30 & 0.764 & -.7339679 & 1.000157 \\ \text { x45 } & .3022004 & .3822778 & 0.79 & 0.429 & -.4470503 & 1.051451 \\ \text { cons } & -.6744898 & .3046939 & -2.21 & 0.027 & -1.271679 & -.0773\end{array}$

Regresión 4: Relación entre desempeño y facturación a las grandes plantas

Número de obs. $=111$

LR chi2(1) $=2.80$

Prob $>$ chi2 $=0.0940$

Log likelihood $=-69.923677$

Pseudo R2 $=0.0197$

\begin{tabular}{rcccccc}
\hline $\mathrm{y} 1$ & Coef. & Std. Err. & $\mathrm{z}$ & $\mathrm{P}>|\mathrm{z}|$ & \multicolumn{2}{c}{$[95 \%$ Conf. Interval] } \\
\hline $\mathrm{x} 31$ & .0059947 & .0036082 & 1.66 & 0.097 & -.0010773 & .0130666 \\
$\mathrm{cons}$ & -.7879011 & .263378 & -2.99 & 0.003 & -1.304112 & -.2716898 \\
\hline
\end{tabular}

Regresión 5: Relación entre inversión y tamaño de la empresa

Número de obs. $=111$

LR chi2(1) $=14.49$

Prob $>$ chi2 $=0.0001$

Log likelihood $=-49.39309$

Pseudo R2 $=0.1279$

\begin{tabular}{rcccccc}
\hline $\mathrm{x} 21$ & Coef. & Std. Err. & $\mathrm{z}$ & $\mathrm{P}>|\mathrm{z}|$ & [95\% Conf. Interval] \\
\hline $\mathrm{x} 11$ & .5619043 & .1586449 & 3.54 & 0.000 & .2509661 & .8728425 \\
cons & -.7505367 & .4499166 & -1.67 & 0.095 & -1.632357 & .1312836 \\
\hline
\end{tabular}

Regresión 6: Estimación del modelo considerando X22

Número de obs. $=111$

LR chi2(5) $=17.59$

Prob $>$ chi2 $=0.0035$

Log likelihood $=-62.532356$

Pseudo R2 $=0.1233$

\begin{tabular}{lllllll}
\hline $\mathrm{y} 1$ & Coef. & Std. Err. & $\mathrm{z}$ & $\mathrm{P}>|\mathrm{z}|$ & \multicolumn{1}{c}{ [95\% Conf. Interval] } \\
\hline x11 & .2630071 & .1561907 & 1.68 & 0.092 & -.043121 & .5691352 \\
x12 & -.0140414 & .0092704 & -1.51 & 0.130 & -.0322111 & .0041283 \\
x21 & .9631064 & .4502769 & 2.14 & 0.032 & .08058 & 1.845633 \\
x22 & .0105609 & .325021 & 0.03 & 0.974 & -.6264686 & .6475904
\end{tabular}


$\begin{array}{lllllll}\text { x31 } & .0059875 & .0038345 & 1.56 & 0.118 & -.0015281 & .013503\end{array}$

$\begin{array}{llllllll}\text { cons } & -2.188304 & .6320034 & -3.46 & 0.001 & -3.427008 & -.9496001\end{array}$ 